\title{
Nucleotide excision repair hotspots and coldspots of UV-induced DNA damage in the human genome
}

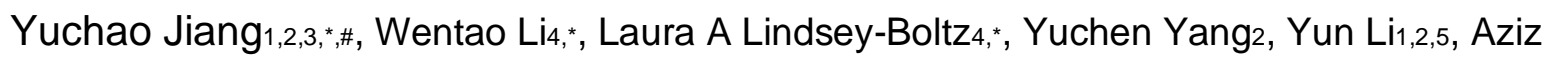

Sancar3,4,\#

1 Department of Biostatistics, Gillings School of Global Public Health, University of North Carolina, Chapel Hill, NC 27599, USA.

2 Department of Genetics, School of Medicine, University of North Carolina, Chapel Hill, NC 27599, USA.

3 Lineberger Comprehensive Cancer Center, University of North Carolina, Chapel Hill, NC 27599, USA.

4 Department of Biochemistry and Biophysics, School of Medicine, University of North Carolina, Chapel Hill, NC 27599, USA.

5 Department of Computer Science, College of Arts and Sciences, University of North Carolina, Chapel Hill, NC 27599, USA.

* These authors contributed equally.

\# To whom correspondence should be addressed. Email: yuchaoj@email.unc.edu, aziz_sancar@med.unc.edu. 


\section{ABSTRACT}

2 We recently developed high-throughput sequencing approaches, eXcision Repair 3 sequencing (XR-seq) and Damage-seq, to generate genome-wide mapping of DNA 4 excision repair and damage formation, respectively, with single-nucleotide resolution. 5 Here, we used time-course XR-seq data to profile UV-induced excision repair dynamics, 6 paired with Damage-seq data to quantify the overall induced DNA damage. We identified

7 genome-wide repair hotspots exhibiting high-level nucleotide excision repair immediately 8 after UV irradiation. We show that such repair hotspots do not result from hypersensitivity

9 to DNA damage, and are thus not damage hotspots. We find that the earliest repair occurs 10 preferentially in promoters and enhancers from open-chromatin regions. The repair 11 hotspots are also significantly enriched for frequently interacting regions and super12 enhancers, both of which are themselves hotspots for local chromatin interactions. 13 Further interrogation of chromatin organization to include DNA replication timing allows 14 us to conclude that early-repair hotspots are enriched for early-replication domains. 15 Collectively, we report genome-wide early-repair hotspots of UV-induced damage, in 16 association with chromatin states and epigenetic compartmentalization of the human 17 genome. 


\section{INTRODUCTION}

19 UV in sunlight is a known mutagen and causative agent of skin cancer that induces DNA lesions such as cyclobutane pyrimidine dimers (CPDs) and pyrimidine-pyrimidone (6-4)

21 photoproducts [(6-4)PPs] $(1,2)$. In humans, both damage types are solely repaired by

22 nucleotide excision repair (excision repair), which removes DNA lesions by dual incisions

23 bracketing modified bases and fills and seals the resulting gap by DNA synthesis and

24 ligation (3). Excision repair consists of two pathways, global repair and transcription-

25 coupled repair $(4,5)$, that differ primarily in the damage recognition step (3-6). For UV-

26 induced DNA damage, CPD repair is highly associated with transcription, specifically with

27 the transcribed strand, while (6-4)PP repair is uniformly distributed throughout the 28 genome (7).

29 Recently, we developed a high-throughput approach, excision repair sequencing

30 (XR-seq), to isolate the oligonucleotides excised by excision repair and subject them to 31 next-generation sequencing (7). This method has allowed for genome-wide mapping of

32 UV-induced excision repair with single-nucleotide resolution in human (7), Lemur (8),

33 mouse (9), Drosophila melanogaster (10), Saccharomyces cerevisiae (11), Escherichia

34 coli (12), and Arabidopsis thaliana (13). We further developed another next-generation

35 sequencing method, Damage-seq (14), to generate genome-wide mapping of UV

36 damage formation with single-nucleotide resolution (15).

Using the combined UV damage maps and repair maps, it has been shown that

38 the induced DNA damage is uniformly distributed throughout the genome and that the

39 overall effect of damage in the genome is primarily driven, not by damage formation, but

40 by repair efficiency $(14,15)$. Indeed, different repair efficiencies across the genome have

41 been previously reported, which are impacted by multiple factors, including transcription,

42 chromatin state and structure, regulatory protein binding to DNA, and posttranscriptional

43 modification of histones (7,15-22). Existing studies, however, have been focused on

44 profiling repair dynamics over a time course $-4 \mathrm{~h}$ for (6-4)PP and $48 \mathrm{~h}$ for CPD. Genomic

45 regions that harbor high-level early repair shortly after damage formation have not been

46 studied, nor have their associated genomic and epigenomic characteristics been

47 systematically explored. 
Here, we performed XR-seq at times as early as 1 min for (6-4)PP and 12 min for CPD following UV irradiation to identify such repair hotspots. We systematically characterized the identified hotspots using additional high-throughput sequencing data that measures DNA damage formation, DNase I hypersensitivity, histone modifications,

52 3D chromatin interactions, and DNA replication timing. These extensive links between

53 chromatin states and organization, cell cycle, DNA damage, and excision repair at

54 specific genomic sites facilitate a better understanding of mutagenesis and

55 carcinogenesis.

\section{RESULTS}

58 Genome-wide profiling of DNA excision repair kinetics through ordered high59 throughput experiments

60 In this work, we present an experimental and analytical framework where we 61 systematically assay DNA excision repair of UV-induced DNA damage over a time course.

62 Figure $1 \mathrm{~A}$ gives an outline of the experimental design. Specifically, we adopted XR-seq 63 (7) to measure repair of (6-4)PPs at $1 \mathrm{~min}, 2 \mathrm{~min}, 5 \mathrm{~min}, 20 \mathrm{~min}, 1 \mathrm{~h}, 2 \mathrm{~h}$, and $4 \mathrm{~h}$ and 64 CPDs at 12 min in normal human skin fibroblasts (NHF1) after 20J/m2 UV treatment. The 65 (6-4)PP XR-seq experiments from $1 \mathrm{~min}$ to $4 \mathrm{~h}$ were all performed with two biological 66 replicates, with correlation coefficients greater than 0.99 between each pair of replicates

67 (Figure S1). Quality control procedures show that all samples have good data quality with 68 expected read length distributions and enrichments of TT and/or TC dinucleotides at the 69 damage sites (Figure S2). To investigate the interplay between DNA damage and repair, 70 we additionally adopted Damage-seq data (15) to profile the two types of UV-induced 71 damage at 0 timepoint after UV irradiation. Refer to the Methods section for details on 72 library preparation, sequencing, and bioinformatic analysis and Table S1 for detailed 73 sample information.

74 The bulk repair pathways and kinetics of these two types of DNA damage are 75 known to be quite different $(23,24)$. The global repair efficiently removes (6-4)PPs, with 76 most of the damage excised within $4 \mathrm{~h}$ of UV treatment. We provide additional empirical 77 evidence that the repair levels of (6-4)PP from the transcribed strand (TS) and the non78 transcribed strand (NTS) are on par across all timepoints (Figure S3A-B). In contrast, 
79 CPDs are recognized primarily in a transcription-coupled manner, and their complete

80 repair requires 12 to $48 \mathrm{~h}$, depending on the UV dose. In our experiment, however, given

81 the sampling timepoint as early as $12 \mathrm{~min}$ for CPDs, transcription-coupled repair has not

82 contributed much, if at all, to the repair of CPDs. Specifically, as shown in Figure S3C,

83 we do not observe a difference between TS and NTS repair at $12 \mathrm{~min}$, which is likely too

84 early for enough polymerases to have stalled and recruited the repair factors (25). This is

85 of key importance so that our analysis is unbiasedly focused on the global repair of (6-

86 4)PP at any timepoints and of CPD at the early 12 min timepoint, without transcription as

87 a confounder. Refer to the Discussion section for more details.

88 In this study, we focus on characterizing global repair kinetics and identifying early-

89 repair hotspots and late-repair coldspots. In vivo excision assay (24) by gel

90 autoradiography at 0 min and 2 min reveals that the primary excision repair products can

91 be detected as early as 2 min after UV treatment (Figure 1B). Using genome-wide repair

92 data by XR-seq, we performed principal component analysis (PCA) (26) on the top 2,000

93 highly variable genes to generate a low-dimensional representation of the (6-4)PP repair

94 data (Figure 1C). Since transcription-coupled repair does not contribute to the repair of

95 (6-4)PPs, the PCA plots do not differ between the TS and NTS repair, except for reverted

96 signs of the eigenvectors. Importantly, a repair trajectory on the principal component

97 space can be reconstructed, which lines up well with the timepoints, suggesting

98 differential repair kinetics over the time course (Figure 1C).

Identification of early-repair hotspots and late-repair coldspots in normal human

102 We developed a computational framework to identify genome-wide early-repair hotspots

103 using time-course XR-seq data. Briefly, we segmented the genome into consecutive bins

104 of $50 \mathrm{bp}$ long and identified bins that showed significantly enriched repair at earlier or later

105 timepoints using a thresholding approach on the downsampled reads (Figure S4). We

106 define such genomic bins as repair hotspots and coldspots, respectively. While such a

107 method is efficient and effective in identifying the top few hundred repair hotspots and

108 coldspots, we additionally adopted a more rigorous Poisson log linear model $(27,28)$ on

109 the read count data for normalization and testing of repair enrichment. The identified 
110 repair hotspots and coldspots show enriched repair levels compared to those as expected 111 under the null (Figure S5). Refer to the Methods section for details.

112 Figure 1D shows the distributions of read counts per genomic bin across all (6113 4)PP samples, and we note enrichment of both early repair at $1 \mathrm{~min}$ and late repair at 4 $114 \mathrm{~h}$, corresponding to repair hotspots and coldspots, respectively. For repair of (6-4)PPs, 115 we identified on the genome-wide scale 175 and 156 repair hotspots from the plus and 116 minus strand (Table S2) and 48 and 57 repair coldspots from the plus and minus strand 117 (Table S3), respectively. For repair of CPDs, we focused on repair hotspots at an early 118 timepoint to negate the global impact of transcription, and identified 99 and 93 repair 119 hotspots from the plus and minus strands, respectively (Table S4). We could not search 120 for intrinsic CPD coldspots, because 30 min after UV irradiation transcription-coupled 121 repair becomes an important contributor to the repair profile.

122 The repair hotspots and coldspots reported are scattered across the entire human 123 genome (Figure S6). XR-seq signals from examples of repair hotspots and coldspots are 124 separated by strand and plotted across all timepoints in Figure 2. We also include 125 epigenomic signals by DNase-seq, ChIP-seq from ENCODE (29), and Damage-seq 126 signals at 0 min after UV treatment (15). Specifically, the XR-seq signals from an example 127 (6-4)PP repair hotspot decrease dramatically from 1 min to $20 \mathrm{~min}$ and can be barely 128 seen at $1 \mathrm{~h}$ (Figure 2A). In contrast, the XR-seq signals at a late-repair coldspot shown in 129 Figure 2B increases over the time course and peaks at $4 \mathrm{~h}$. Another representative CPD 130 repair hotspot at 12 min is shown in Figure 2C.

131 We next performed sequence context analysis using all reads that are mapped to 132 the repair hotspots and coldspots, respectively. We trimmed the reads to be 15 bp long 133 centering at the damage sites and calculated strand-specific nucleotide frequencies in 134 repair hotspots, coldspots, and randomly picked spots. Interestingly, we identified an 135 enrichment of cytosine in the flanking regions of the damage/repair sites for both repair 136 hotspots and coldspots (Figure S7). Motif analysis by the MEME suite (30) confirmed the 137 enrichment of cytosine adjacent to the repair sites, which are themselves enriched with 138 canonical sequences of CTCA for (6-4)PP and TT for CPD (Table S5). While it has been 139 previously shown that active transcription factor binding sites have decreased levels of 140 excision repair (21), here we report a preference for cytosine at both early and late 
141 timepoints, which have increased levels of repair compared to null regions. Notably, such

142 cytosine enrichment in the flanking regions of the repair sites is not due to sequence

143 context bias, as we show in Figure S8 that not all genomic regions enriched for cytosines

144 are enriched for excision repair. While an interesting observation, the underlying

145 biological mechanisms need further investigation.

\section{Early-repair hotspots are not hypersensitive to DNA damage}

148 A recent study assayed DNA damage by next-generation sequencing and reported a total 149 of 153 hyper-hotspots acquiring CPDs much more frequently than the genomic average 150 in primary human fibroblasts (31). Of these damage hotspots, 83 are from the plus strand 151 and 74 are from the minus strand, each having at least five recurrent sequence reads 152 (31). To investigate whether the identified repair hotspots simply result from increased 153 levels of DNA damage, we first intersected the reported damage hotspots from Premi et 154 al. (31) with the 99 and 93 CPD repair hotspots from the plus and minus strand that we 155 identified. We found that none of our identified repair hotspots overlapped with the 156 reported damage hotspots.

157 To further confirm and replicate this seemingly striking result, we analyzed 158 genome-wide CPD DNA damage data generated by our previously developed Damage159 seq protocol (15), to quantify damage levels at 0 timepoint after UV irradiation with single160 nucleotide resolution. After stringent quality control procedures (refer to the Methods 161 section for details), we identified 91 and 78 CPD damage hotspots from the plus and 162 minus strand, respectively, each having at least ten mapped reads (Table S6). Notably, 163 these Damage-seq hotspots are shown to be enriched for heterochromatin and repressed 164 regions (Figure S9), which is concordant with previous reports $(32,33)$. Importantly, none 165 of the CPD damage hotspots, identified from this parallel Damage-seq platform, overlap 166 with the repair hotspots.

167 In addition, we compared the DNA damage levels for (6-4)PP and CPD from three 168 independent sequencing technologies - Damage-seq (15), adductSeq (31), and CPD169 seq (19) - at our identified hotspots and coldspots against those from randomly sampled 170 regions along the genome. To account for the sparse sampling when measuring DNA 171 damage by next-generation sequencing, we also extend the regions corresponding to the 
172 repair hotspots, coldspots, and random spots at both ends for 20bp and 500bp, 173 respectively. Our results, shown in Figure 3, suggest that there is no significant difference

174 in the damage levels between the three repair categories (hotspot, coldspot, and random 175 spot). The zoom-in and zoom-out views of three example repair hotspots and coldspots 176 in Figure 2 also suggest that the Damage-seq reads are uniformly distributed in the 177 flanking regions. Previous results have demonstrated that the UV-induced DNA damage 178 is indeed virtually uniform across the entire human genome, while repair is affected by 179 chromatin states, transcription factor binding, etc., in a manner dependent on the type of 180 DNA damage (15). While we note that the shallow depth of coverage of Damage-seq can 181 be a limiting factor (refer to the Discussion section for details), our results validate our 182 conclusion that the identified repair hotspots are not damage hotspots.

Early-repair hotspots are enriched for promoters and enhancers in open chromatin 185 regions

186 The packaging of DNA into chromatin can hinder the access of repair proteins and affect 187 the repair efficiency $(16,17)$ and specific histone modifications are associated with 188 different functional and cytological chromatin states. To investigate the relationship 189 between the identified repair hotspots/coldspots and the epigenomic markers, we used 190 publicly available DNase-seq data and histone modification ChIP-seq data for an adult 191 human fibroblast cell line (NHLF) from ENCODE (29). Our results suggest that the earliest 192 repair occurs preferentially in active and open-chromatin regions. Specifically, chromatin 193 accessibility by DNase-seq is significantly higher in repair hotspots than in random 194 genomic regions, and it is significantly lower in repair coldspots (Figure 4). For histone 195 modifications, repair hotspots have significantly higher ChIP-seq signals compared to 196 random spots for activation markers, including H3K4me1, H3K4me3, and H3K27ac 197 (Figure 4).

198 We adopted the segmented chromatin states by chromHMM (34) to annotate the 199 repair hotspots for (6-4)PP and CPD and found a significant enrichment of active 200 promoters and enhancers (Figure 5A), which are characterized by nucleosome loss and 201 open chromatin. Genome-wide repair kinetics inferred across timepoints confirm that 202 enhancers are repaired at earlier timepoints and that repressed/heterochromatin regions 
203 are repaired at later timepoints (Figure S10). Additionally, we performed genome-wide

204 annotations for CpG islands and genic/intergenic regions. Our results suggest that the 205 repair hotspots are enriched for inter CpG regions (i.e., depleted for CpG islands) (Figure 206 5B) and intronic regions (Figure 5C), both of which are enriched for AT and presumably 207 result in more repair. Notably, the number of identified repair coldspots is too low to 208 generate reproducible annotation results between the replicates.

210 Early-repair hotspots are enriched for frequently interacting regions (FIREs) and 211 super-enhancers

212 With the genome-wide enrichment of active promoters and enhancers in open chromatin 213 regions of the detected repair hotspots, we hypothesize that the 3D genome structure 214 contributes to the observed differential repair kinetics between different regions of the 215 genome. To test this hypothesis, we sought to interrogate the publicly available Hi-C data 216 of human fibroblast cell line IMR90 (35,36). Specifically, after quality control procedures 217 and data normalization, we profiled frequently interacting regions (FIREs) using 218 FIREcaller (37). After overlapping the repair hotspots and coldspots with the called FIREs

219 (Table S7A), we found that a significantly higher proportion of repair hotspots overlap with 220 FIREs - 23.16\% and $11.76 \%$ for (6-4)PP and CPD, respectively - compared to a genome 221 average of $6.93 \%$ based on the profiled FIREs (Figure 6A). Conversely, the overlapping 222 proportion of (6-4)PP repair coldspots is $3.23 \%$, significantly lower than the genome 223 average (Figure 6A).

224 FIREs have been previously reported to be enriched for super-enhancers (38). We 225 have demonstrated that the repair hotspots are enriched for both FIREs and enhancers. 226 We also observed that, across many cases, multiple enhancers that overlapped with the 227 repair hotspots were from the same genomic regions (Figure S11). As such, we 228 hypothesize that the repair hotspots are also enriched for super-enhancers and thus 229 additionally adopted a list of previously annotated super-enhancers in the human 230 fibroblasts (39) (Table S7B). We found that, compared to a genome-wide average of $2312.05 \%$, the early-repair hotspots are indeed enriched for super-enhancers (5.14\% and $2324.69 \%$ for (6-4)PP and CPD repair hotspots, respectively), while none of the repair 233 coldspots overlap with super-enhancers (0\% for (6-4)PP repair coldspot) (Figure 6B). 
In addition, we called significant interactions based on the $\mathrm{Hi}-\mathrm{C}$ contact matrix using the Fit-Hi-C method (40) (Table S7C) and showed that early-repair hotspots also overlap with a significantly higher number of significant interactions (Figure 6C). Refer to the Methods section for details on data analysis. The overlapping information of the called

238 repair hotspots and coldspots with the profiled FIREs, super-enhancers, and significant 239 chromatin interactions are included in Table S8. Figure 6D illustrates the loop interactions 240 of two identified repair hotspots. Notably, these two hotspots also overlap with both FIREs

241 and super-enhancers. These results collectively provide a global picture of genetic 242 regulation of repair kinetics via 3D genome structures.

\section{Early-repair hotspots are enriched for early-replication domains}

245 We next set out to test the relationship between the repair kinetics and replication timing.

246 We used the genome-wide Repli-Seq data of the human fibroblast cell line IMR90, which

247 maps high-resolution DNA replication patterns with respect to both cell-cycle time and 248 genomic position (41). A recent re-analysis of the data using a deep-learning model (42) 249 segmented the genome into different replication domains, including the early-replication 250 domain (ERD), late-replication domain (LRD), up-transition zone (UTZ), and down251 transition zone (DTZ). Refer to Table S9 for genome-wide segmentation results. When 252 overlapping the identified repair hotspots with the segmented replication domains, we find 253 that early-repair hotspots are also significantly enriched for early-replication domains 254 (Figure 7). While replication time has been shown to be correlated with chromatin 255 accessibility (41), here we have demonstrated a potential cell-cycle effect on DNA 256 excision repair. That is, regions that are duplicated early also tend to be repaired early, 257 and this has been shown to result in mutagenesis asymmetries (17) - we further elaborate 258 such implications in the Discussion section.

260 Profiling gene-level repair kinetics in normal human fibroblasts

261 So far, our analysis has been conducted on the whole-genome level. Identifying dynamic 262 changes at individual gene levels can provide important insights about genes and 263 pathways that exhibit significant changes in repair dynamics (43). We calculated strand264 specific reads per kilobase per million reads (RPKM) for all the genes in the human 
265 genome. RPKMs across nine genes associated with circadian rhythm (e.g., CLOCK, 266 BMAL1, PER1, CRY1) and excision repair (e.g., XPA, XPC, CSA, CSB) present different 267 repair dynamics over the time course (Figure S12). We adopted Trendy (44) to perform 268 segmented regression analysis (Table S10) on the ordered XR-seq data. We focused on 269 significant genes with adjusted $R^{2}$ greater than 0.8 from the regression analysis and 270 identified 3,017 significant genes with fitted trend as "down," 3,496 significant genes with 271 fitted trend as "up-down," 1,024 significant genes with fitted trend as "up," and 1,452 272 significant genes with fitted trend as "down-up" (Figure S13). The number of genes that 273 show the "down" trend is two-fold higher than the number of genes that show the "up" 274 trend, which is consistent with our previous observation - there are more repair hotspots 275 than coldspots. For these four groups of significant genes, we further performed gene 276 ontology (GO) enrichment analysis using PANTHER (45). For genes with the "down" and 277 "up-down” trend, GO biological processes are enriched for a large number of biological 278 regulations, including regulation of the metabolic process, gene expression, 279 developmental process, cell cycle, and cell death, etc. (Table S11A-B). On the other hand, 280 there are no obvious GO enrichment terms for the "up" or "down-up" genes (Table S11C281 D).

\section{DISCUSSION}

284 We adopted time-course XR-seq data to detect genome-wide repair hotspots and 285 coldspots and integrated additional omics data from various next-generation sequencing 286 platforms to investigate the relationship between DNA damage, excision repair, 287 epigenomic markers, 3D genome organization, and replication timing. To our best 288 knowledge, this, for the first time, demonstrates the connection between repair kinetics 289 and chromatin organization (46) with high resolution in human cell lines. The identified 290 repair hotspots can serve as additional functional genomic features in studies of the 291 human genome at the multidimensional level. We believe the framework and multi-omics 292 data we present here can be useful to scientists both in the fields of chromatin dynamics 293 and those interested in determinants of repair rates in the human genome. Our approach 294 is applicable to studying the response to other types of DNA damage, including that 
295 induced by drugs used in chemotherapy and thus has the potential for informing new 296 therapeutic strategies.

297 Using paired XR-seq and Damage-seq data, we provided additional empirical 298 evidence that the damage levels in the regions of the repair hotspots were not enriched, 299 in concordance with previous reports $(14,15)$. Therefore, we attributed the observed 300 differences in repair patterns to heterogeneous repair efficiencies and not to damage 301 formation. It is, however, noteworthy that the depth of coverage by Damage-seq is an 302 order of magnitude lower than that by XR-seq. Specifically, the genome-wide average 303 total number of reads between the two replicates for (6-4)PP Damage-seq is 20.5 million, 304 while that average for (6-4)PP XR-seq is 181.8 million (Table S1). As such, it is possible 305 that we do not have enough sequencing depth to detect Damage-seq hotspots with high sensitivity. The fact that a good proportion of Damage-seq reads get mapped to

307 heterochromatin regions further complicates analysis and lowers detection power. This 308 issue also persists for the recently developed adductSeq and FreqSeq, where a Poisson 309 model was adopted with a genomic average rate of 0.07 reads per pyrimidine dinucleotide 310 in fibroblasts (31). A Poisson distribution with a mean parameter under the null much less 311 than one could potentially suffer from overdispersion and inflated false positive rates. 312 Targeted Damage-seq offers a solution for both discovery and validation. However, as 313 with targeted DNA sequencing, the data can be extremely noisy due to targeting, 314 amplification, and sequencing biases and artifacts (47).

315 Using the genome-wide profile of DNA replication timing, we demonstrate that the 316 early-repair hotspots also tend to reside in early-replication domains. The inevitable effect 317 of replication on gene dosage and copy number could also have regulatory consequences 318 (48). Genes/regions that are duplicated early will be present at twice the copy number of 319 late replicating domains for most of the duration of $S$ phase, increasing both the DNA 320 amount (as sister chromatids) and the transcriptional output. The ability to perform 321 excision repair for a greater fraction of the cell cycle has been shown to result in lower 322 mutation rates in the early-replication domains $(49,50)$.

323 UV-induced DNA damage and excision repair have been linked with mutagenesis 324 and carcinogenesis. Recent studies have shown that mutation hotspots exhibit strong 325 increases in CPD formation efficacy (51-53) and that excision repair is attenuated in 
326 transcription factor binding sites, leading to elevated somatic mutation rates $(19,21)$. In

327 terms of repair kinetics, Adar et al. (17) showed that globally late-repaired regions are

328 associated with a higher level of cancer-linked mutations. To further investigate this in our

329 study context, we quantified the rates of somatic point mutations associated with

330 melanoma (1) in the repair hotspots and coldspots. However, due to the low number of

331 hotspots and coldspots, as well as their small genomic lengths, we found very few

332 overlapped with mutations and were thus underpowered to test for the differences in

333 mutation rates between different repair categories.

334 While the repair of (6-4)PP is predominantly carried out by global repair,

335 transcription-coupled repair plays an essential role in removing the more abundant and

336 less helix-distorting CPD adducts. In this study, we have intentionally focused on profiling

337 global repair hotspots for both (6-4)PP and CPD at early timepoints without the effect of

338 transcription-coupled repair. Genes exhibit both transcriptional dynamics over a time

339 course and biological fluctuations due to transcriptional bursting (54) at the same

340 timepoint. This confounds and complicates the analysis. To disentangle the effects of

341 global repair and transcriptional-coupled repair in identifying repair hotspots is an

342 unsolved yet challenging problem.

344 METHODS

345 Experimental methods

346 Cell culture and UV irradiation

347 Human NHF1 cells were obtained from W.K. Kaufmann (University of North Carolina, 348 Chapel Hill) (55) and cultured in Dulbecco's Modified Eagle Medium (DMEM) with 10\% 349 FBS at $37^{\circ} \mathrm{C}$ in a $5 \% \mathrm{CO}_{2}$ humidified chamber. For (6-4)PP XR-seq at 1 min and 2 min 350 timepoints, UV irradiation was performed as previously described $(7,56)$. Briefly, the $80 \%$ 351 confluent NHF1 cells in one petri dish were irradiated for $20 \mathrm{~s}$ under a $250 \mathrm{~nm}$ UV lamp $352\left(1 \mathrm{~J} / \mathrm{m}_{2} / \mathrm{s}\right)$ after removing the culture medium. $37^{\circ} \mathrm{C}$ DMEM with $10 \%$ FBS medium was 353 immediately added into the petri dish, then the medium was poured off and the petri dish 354 was put on ice promptly at the end of 1 min or 2 min after UV irradiation. The time count 355 starts from the end of $20 \mathrm{~s}$ UV irradiation and ends at the timepoint when the petri dish is 356 put on ice. The cells were washed one time with ice-cold PBS before being harvested by 
357 a cell scraper in $10 \mathrm{ml}$ ice-cold PBS. In each replicate of (6-4)PP XR-seq experiment, 50 358 and 30 petri dishes $(150 \mathrm{~mm} \times 15 \mathrm{~mm})$ containing NHF1 cells were treated one by one at

$3591 \mathrm{~min}$ and $2 \mathrm{~min}$ timepoints, respectively. Cell culture, UV treatment, and library 360 preparation for (6-4)PP XR-seq at $5 \mathrm{~min}, 20 \mathrm{~min}, 1 \mathrm{~h}, 2 \mathrm{~h}$, and $4 \mathrm{~h}$, and CPD XR-seq at 36112 min were performed in previous studies $(7,10,17)$. For in vivo excision assay, UV 362 irradiation was performed as aforementioned, and 10 and 5 petri dishes $(150 \mathrm{~mm} \times 15 \mathrm{~mm})$ 363 containing NHF1 cells were used at 0 min and 2 min timepoints respectively.

\section{Excision assay}

366 The in vivo excision assay was performed as described (24,57). Following UV irradiation, 367 the excision products were isolated by gentle cell lysis and nonchromatin fraction 368 separation and purified by TFIIH immunoprecipitation. The purified excision products 369 were then 3' radiolabeled by terminal deoxynucleotidyl transferase and [a-32P]-3'-dATP, 370 and resolved in a $10 \%$ denaturing acrylamide gel. Ten and five petri dishes $(150 \mathrm{~mm} \times$ $37115 \mathrm{~mm}$ ) of NHF1 cells were used at 0 min and 2 min, respectively.

\section{$X R$-seq library preparation and sequencing}

374 XR-seq libraries were prepared as described in the previous protocol (57). Briefly, the excision products were isolated by TFIIH immunoprecipitation following gentle cell lysis and non-chromatin fraction separation, and ligated with adaptors. The ligated excision products were then further purified by immunoprecipitation with anti-(6-4)PP antibody and repaired by (6-4)PP photolyase before the library amplification by PCR. Libraries were sequenced on an Illumina HiSeq 4000 platform.

Data collection

382 (6-4)PP XR-seq data at $5 \mathrm{~min}, 20 \mathrm{~min}, 1 \mathrm{~h}, 2 \mathrm{~h}$, and $4 \mathrm{~h}$ were downloaded from the Gene 383 Expression Omnibus (GEO) with accession numbers GSE67941 (7) and GSE76391 (17). 384 CPD XR-seq data at 12 min were downloaded from GEO with accession number 385 GSE138846 (10). CPD and (6-4)PP damage data of NHF1 by Damage-seq were 386 downloaded from GEO with accession number GSE98025 (15); CPD damage data of 387 NHF1 by CPD-seq were downloaded from GEO with accession number GSM2772322 
and GSM2772323 (19); CPD damage data of human primary fibroblast by adductSeq were downloaded from GEO with accession number GSM4073616 and GSM4073634 (31). Hyper-hotspots for UV-induced CPD damage in primary human fibroblasts were downloaded from Premi et al. (31). NHDF H3K4me1 (ENCODE Data Coordination Center

392 accession number ENCSR000ARV), H3K4me3 (accession number ENCSR000DPR), 393 H3K27ac (accession number ENCSR000APN), H3K27me3 (accession number 394 ENCSR000APO), H3K9me3 (accession number ENCSR000ARX), and DNase-seq 395 (accession number ENCSR000EMP) data were downloaded from the ENCODE portal 396 (29). NHLF chromatin state segmentation results by chromHMM were downloaded from 397 UCSC accession number wgEncodeEH000792 (34). Hi-C data of IMR90 were 398 downloaded from GEO with accession number GSE43070 (35) and from 399 https://bioconductor.org/packages/HiCDataHuman/MR90/ (36). The list of annotated 400 super-enhancers in IMR90 was downloaded from the Roadmap Epigenomics Consortium 401 (39). Genomic categories of replication timing from Repli-Seq data of IMR90 (41) were 402 downloaded from GSE53984 (42).

\section{Bioinformatic and statistical analysis}

\section{$405 \quad$ XR-seq bioinformatic pre-processing}

406 For XR-seq, cutadapt (58) was used to trim reads with adaptor sequence 407 TGGAATTCTCGGGTGCCAAGGAACTCCAGTNNNNNNACGATCTCGTATGCCGTCT 408 TCTGCTTG at the 3' end and discard untrimmed reads. BWA (59) was used for 409 alignment of single-end short reads. Unmapped reads and reads that map to multiple 410 locations with the same alignment quality were removed using Samtools (60). Post411 alignment filtering steps were adopted using Rsamtools 412 (http://bioconductor.org/packages/Rsamtools/). Specifically, if multiple reads share the 413 same 5' and 3' end coordinates, we keep only one to perform deduplication. We also only 414 keep reads that have mapping quality greater than 20 and are of lengths $21 \mathrm{bp}$ to $31 \mathrm{bp}$.

417 Reads from the TS and NTS strands were separated using known gene annotations for 418 hg19 by ENSEMBL. We use RPKM for within-sample normalization for the XR-seq data. 
419 To perform gene-level quantification and downstream analysis including segmented 420 regression, we adopted a stringent quality control procedure and only retained genes that:

421 (i) had at least ten TT or TC dinucleotides from either TS or NTS; (ii) were less than 300Kb;

422 and (iii) had at least ten reads in total across all XR-seq samples. In addition, we took the 423 ratio of the reads from the TS and the NTS [TS/(TS+NTS)] to remove biases and artifact 424 that are shared between the two DNA strands, i.e., library size, gene length, and other 425 gene-specific biases, such as sequencing bias and antibody pulldown efficiency, etc. The 426 ratio is bound between 0 and 1 and sheds light upon how transcription-coupled repair and 427 global repair interplay (Figure S3). Identification of repair hotspots and coldspots

430 We started by segmenting the human reference genome into consecutive bins of $50 \mathrm{bp}$ 431 long. We then calculated the observed depth of coverage per bin by XR-seq, separating 432 the plus-strand reads (+) and the minus-strand reads (-). To mitigate the effect of library 433 size/sequencing depth, we downsampled the reads in each sample to 7.7 million without 434 replacement. To identify repair hotspots and coldspot, we set a threshold on the number 435 of read counts per genomic bin in the $1 \mathrm{~min}$ and $4 \mathrm{~h}$ samples. Specifically, to identify (6436 4)PP repair hotspots, we require at least 15 reads mapped in both replicates at $1 \mathrm{~min}$ and 437 at most 5 reads mapped in both replicates at $4 \mathrm{~h}$. The read count threshold is relaxed for 438 the identification of coldspots, which have a smaller number compared to the hotspots. 439 For CPD repair, to avoid complicatedness due to transcription-coupled repair at later 440 timepoints, we focused on CPD repair hotspots only.

441 In addition to the thresholding approach, we adopted a more rigorous cross442 sample Poisson log linear model $(27,28)$ for data normalization. Specifically, we denote $443 \quad Y$ as the observed repair matrix, with row $i$ corresponding to the $i$ th genomic bin and 444 column $j$ corresponding to the $j$ th sample. The "null" model, which reflects the expected 445 coverage when there is no biologically relevant repair enrichment, is

$$
Y_{i j} \sim \operatorname{Poisson}\left(\lambda_{i j}\right), \quad \lambda_{i j}=N_{j} \beta_{i} f_{j}\left(T C_{i}\right),
$$

447 where $N_{j}$ is the total number of mapped reads for sample $j$ (fixed for downsampled data),

$448 \beta_{i}$ reflects the bin-specific bias due to library preparation and sequencing bias, and $449 f_{j}\left(T C_{i}\right)$ is the sample-specific bias due to TC (thymine and cytosine) content for 
450 damage/repair. The goal of fitting the null model to the data is to estimate the various 451 sources of biases, which can then be used for normalization. We adopt a robust iterative 452 maximum-likelihood algorithm (28) for estimating the parameters of the null model. Plus 453 and minus strands are analyzed separately.

$454 \quad$ Given a first-pass of the calling algorithms, we identified strong repair hotspots in 455 pericentromeric regions, which were collapsed repeats annotated as unique sequences 456 in the reference genome (e.g., ribosomal DNA (9)). It is important to exclude artifacts as 457 stringently as possible, and thus we undertook an additional quality control step. "Blacklist" 458 bins, including segmental duplication regions 459 (http://humanparalogy.gs.washington.edu/build37/data/GRCh37GenomicSuperDup.tab), 460 gaps in reference assembly from telomere, centromere, and/or heterochromatin regions 461 (https://gist.github.com/leipzig/6123703), and repeating elements by RepeatMasker 462 (https://genome.ucsc.edu/cgi-bin/hgTrackUi?g=rmsk) are masked in downstream 463 analysis.

\section{Hi-C data analysis}

466 We adopted the Hi-C data of human fibroblast cell line IMR90 $(35,36)$ to investigate the 467 relationship between identified repair hotspots and the 3D genome structure. We took the 468 raw contact matrix with $40 \mathrm{~kb}$ resolution as input and detected FIREs, which play 469 important roles in transcriptional regulations, across the entire genome using FIREcaller 470 (37). To further investigate whether these repair hotspots are involved in functional 471 chromatin looping between regulatory elements and their target genes, we adopted the 472 Fit-Hi-C approach (40) to identify long-range chromatin interactions on all $40 \mathrm{~kb}$ bin pairs 473 within a maximal 3 MB region. The interactions with $p$-value $<2.31$ - 11 were considered 474 as statistically significant (61).

476 DATA AND CODE AVAILABILITY

477 The data reported in this paper have been deposited in GEO with accession number 478 GSE148303. Scripts used in this paper are available at 479 https://github.com/yuchaojiang/damage_repair. 
COMPETING INTEREST STATEMENT

482 The authors declare no competing interests.

\section{ACKNOWLEDGEMENTS}

485 This work was supported by NIH Grants R35 GM118102 (to A.S.), R01 ES027255 (to A.S.), R35 GM138342 (to Y.J.), K99 ES030015 (to W.L.), and a pilot award from the UNC Computational Medicine Program (to Y.J.). We thank the Sancar Lab members and Drs. Sheera Adar, Ming Hu, and Jeremy Simon for useful comments and feedback.

\section{AUTHOR CONTRIBUTIONS}

AS envisioned and initiated the study, while LW and LAL-B performed the experiments.

YJ devised the analytical framework, and all authors executed the data analysis. YJ wrote the manuscript, with contributions from WL on experimental methods. The manuscript was further edited and approved by all authors.

\section{REFERENCES}

1. Hodis, E., Watson, I.R., Kryukov, G.V., Arold, S.T., Imielinski, M., Theurillat, J.P., Nickerson, E., Auclair, D., Li, L., Place, C. et al. (2012) A landscape of driver mutations in melanoma. Cell, 150, 251-263.

2. Martincorena, I., Roshan, A., Gerstung, M., Ellis, P., Van Loo, P., McLaren, S., Wedge, D.C.,

3. Sancar, A. (2016) Mechanisms of DNA repair by photolyase and excision nuclease (Nobel

4. Hanawalt, P.C. and Spivak, G. (2008) Transcription-coupled DNA repair: two decades of

5. Mellon, I., Spivak, G. and Hanawalt, P.C. (1987) Selective removal of transcription-blocking

6. Kusakabe, M., Onishi, Y., Tada, H., Kurihara, F., Kusao, K., Furukawa, M., Iwai, S., Yokoi,

7. Hu, J., Adar, S., Selby, C.P., Lieb, J.D. and Sancar, A. (2015) Genome-wide analysis of human global and transcription-coupled excision repair of UV damage at singlenucleotide resolution. Genes Dev, 29, 948-960. 
8. $\quad$ Akkose, U., Kaya, O., Lindsey-Boltz, L., Karagoz, Z., Brown, A.D., Larsen, P.A., Yoder, A.D., Sancar, A. and Adebali, O. (2020) Comparative analyses of two primate species diverged by more than 60 million years show different rates but similar distribution of genomewide UV repair events. bioRxiv.

9. Yang, Y., Hu, J., Selby, C.P., Li, W., Yimit, A., Jiang, Y. and Sancar, A. (2019) Singlenucleotide resolution analysis of nucleotide excision repair of ribosomal DNA in humans and mice. J Biol Chem, 294, 210-217.

10. Deger, N., Yang, Y., Lindsey-Boltz, L.A., Sancar, A. and Selby, C.P. (2019) Drosophila, which

12. Adebali, O., Chiou, Y.Y., Hu, J., Sancar, A. and Selby, C.P. (2017) Genome-wide lacks canonical transcription-coupled repair proteins, performs transcription-coupled repair. J Biol Chem, 294, 18092-18098.

13. Oztas, O., Selby, C.P., Sancar, A. and Adebali, O. (2018) Genome-wide excision repair in

11. Li, W., Adebali, O., Yang, Y., Selby, C.P. and Sancar, A. (2018) Single-nucleotide resolution dynamic repair maps of UV damage in Saccharomyces cerevisiae genome. Proc Natl Acad Sci U S A, 115, E3408-E3415. transcription-coupled repair in Escherichia coli is mediated by the Mfd translocase. Proc Natl Acad Sci U S A, 114, E2116-E2125. Arabidopsis is coupled to transcription and reflects circadian gene expression patterns. Nat Commun, 9, 1503.

14. Hu, J., Lieb, J.D., Sancar, A. and Adar, S. (2016) Cisplatin DNA damage and repair maps of the human genome at single-nucleotide resolution. Proc Natl Acad Sci U S A, 113, 1150711512.

17. Adar, S., Hu, J., Lieb, J.D. and Sancar, A. (2016) Genome-wide kinetics of DNA excision

18. Mao, P., Smerdon, M.J., Roberts, S.A. and Wyrick, J.J. (2016) Chromosomal landscape of

19. Mao, P., Brown, A.J., Esaki, S., Lockwood, S., Poon, G.M.K., Smerdon, M.J., Roberts, S.A. predicts cancer mutagenesis. EMBO J, 36, 2829-2843. 
21. Sabarinathan, R., Mularoni, L., Deu-Pons, J., Gonzalez-Perez, A. and Lopez-Bigas, N. (2016) Nucleotide excision repair is impaired by binding of transcription factors to DNA. Nature, 532, 264-267.

22. Poulos, R.C., Thoms, J.A.I., Guan, Y.F., Unnikrishnan, A., Pimanda, J.E. and Wong, J.W.H. (2016) Functional Mutations Form at CTCF-Cohesin Binding Sites in Melanoma Due to Uneven Nucleotide Excision Repair across the Motif. Cell reports, 17, 2865-2872.

23. Reardon, J.T. and Sancar, A. (2003) Recognition and repair of the cyclobutane thymine dimer, a major cause of skin cancers, by the human excision nuclease. Genes Dev, 17, 2539-2551.

24. Hu, J., Choi, J.H., Gaddameedhi, S., Kemp, M.G., Reardon, J.T. and Sancar, A. (2013) Nucleotide excision repair in human cells: fate of the excised oligonucleotide carrying DNA damage in vivo. J Biol Chem, 288, 20918-20926.

25. Deger, N., Yang, Y., Lindsey-Boltz, L.A., Sancar, A. and Selby, C.P. (2019) Drosophila, which lacks canonical transcription-coupled repair proteins, performs transcription-coupled repair. Journal of Biological Chemistry, 294, 18092-18098.

26. Jolliffe, I.T. and Cadima, J. (2016) Principal component analysis: a review and recent

27. Witten, D.M. (2011) Classification and clustering of sequencing data using a Poisson

28. Jiang, Y., Oldridge, D.A., Diskin, S.J. and Zhang, N.R. (2015) CODEX: a normalization and copy number variation detection method for whole exome sequencing. Nucleic Acids Res, 43, e39.

29. Consortium, E.P. (2012) An integrated encyclopedia of DNA elements in the human genome. Nature, 489, 57-74.

30. Bailey, T.L., Boden, M., Buske, F.A., Frith, M., Grant, C.E., Clementi, L., Ren, J., Li, W.W.

31. Premi, S., Han, L., Mehta, S., Knight, J., Zhao, D., Palmatier, M.A., Kornacker, K. and Brash,

32. Han, C., Srivastava, A.K., Cui, T., Wang, Q.E. and Wani, A.A. (2016) Differential DNA lesion

33. Hauer, M.H. and Gasser, S.M. (2017) Chromatin and nucleosome dynamics in DNA

34. Ernst, J. and Kellis, M. (2012) ChromHMM: automating chromatin-state discovery and and Noble, W.S. (2009) MEME SUITE: tools for motif discovery and searching. Nucleic Acids Res, 37, W202-208.

D.E. (2019) Genomic sites hypersensitive to ultraviolet radiation. Proc Natl Acad Sci U S A, 116, 24196-24205. formation and repair in heterochromatin and euchromatin. Carcinogenesis, 37, 129-138. damage and repair. Genes Dev, 31, 2204-2221.

characterization. Nat Methods, 9, 215-216. 
35. Jin, F., Li, Y., Dixon, J.R., Selvaraj, S., Ye, Z., Lee, A.Y., Yen, C.A., Schmitt, A.D., Espinoza, C.A. and Ren, B. (2013) A high-resolution map of the three-dimensional chromatin interactome in human cells. Nature, 503, 290-294.

36. Dixon, J.R., Selvaraj, S., Yue, F., Kim, A., Li, Y., Shen, Y., Hu, M., Liu, J.S. and Ren, B. (2012) Topological domains in mammalian genomes identified by analysis of chromatin interactions. Nature, 485, 376-380.

37. Crowley, C., Yang, Y., Qiu, Y., Hu, B., Won, H., Ren, B., Hu, M. and Li, Y. (2019) FIREcaller: an $\mathrm{R}$ package for detecting frequently interacting regions from $\mathrm{Hi}-\mathrm{C}$ data. bioRxiv, 619288.

38. Schmitt, A.D., Hu, M., Jung, I., Xu, Z., Qiu, Y., Tan, C.L., Li, Y., Lin, S., Lin, Y., Barr, C.L. et al. (2016) A Compendium of Chromatin Contact Maps Reveals Spatially Active Regions in the Human Genome. Cell Rep, 17, 2042-2059.

39. Consortium, R.E., Kundaje, A., Meuleman, W., Ernst, J., Bilenky, M., Yen, A., HeraviMoussavi, A., Kheradpour, P., Zhang, Z., Wang, J. et al. (2015) Integrative analysis of 111 reference human epigenomes. Nature, 518, 317-330.

40. Ay, F., Bailey, T.L. and Noble, W.S. (2014) Statistical confidence estimation for Hi-C data reveals regulatory chromatin contacts. Genome Res, 24, 999-1011.

41. Hansen, R.S., Thomas, S., Sandstrom, R., Canfield, T.K., Thurman, R.E., Weaver, M., Dorschner, M.O., Gartler, S.M. and Stamatoyannopoulos, J.A. (2010) Sequencing newly replicated DNA reveals widespread plasticity in human replication timing. Proc Natl Acad Sci U S A, 107, 139-144.

42. Liu, F., Ren, C., Li, H., Zhou, P., Bo, X. and Shu, W. (2016) De novo identification of replication-timing domains in the human genome by deep learning. Bioinformatics, 32, 641-649.

43. Li, W., Liu, W., Kakoki, A., Wang, R., Adebali, O., Jiang, Y. and Sancar, A. (2019) Nucleotide excision repair capacity increases during differentiation of human embryonic carcinoma cells into neurons and muscle cells. J Biol Chem, 294, 5914-5922.

44. Bacher, R., Leng, N., Chu, L.F., Ni, Z., Thomson, J.A., Kendziorski, C. and Stewart, R. (2018) Trendy: segmented regression analysis of expression dynamics in high-throughput ordered profiling experiments. BMC Bioinformatics, 19, 380.

45. Thomas, P.D., Campbell, M.J., Kejariwal, A., Mi, H., Karlak, B., Daverman, R., Diemer, K., Muruganujan, A. and Narechania, A. (2003) PANTHER: a library of protein families and subfamilies indexed by function. Genome Res, 13, 2129-2141.

46. van Eijk, P., Nandi, S.P., Yu, S., Bennett, M., Leadbitter, M., Teng, Y. and Reed, S.H. (2019) Nucleosome remodeling at origins of global genome-nucleotide excision repair occurs at the boundaries of higher-order chromatin structure. Genome Res, 29, 74-84.

47. Jiang, Y., Wang, R., Urrutia, E., Anastopoulos, I.N., Nathanson, K.L. and Zhang, N.R. (2018) CODEX2: full-spectrum copy number variation detection by high-throughput DNA sequencing. Genome Biol, 19, 202. 
48. Rhind, N. and Gilbert, D.M. (2013) DNA replication timing. Cold Spring Harb Perspect Biol, 5, a010132.

49. Stamatoyannopoulos, J.A., Adzhubei, I., Thurman, R.E., Kryukov, G.V., Mirkin, S.M. and Sunyaev, S.R. (2009) Human mutation rate associated with DNA replication timing. Nat Genet, 41, 393-395.

50. Lang, G.I. and Murray, A.W. (2011) Mutation rates across budding yeast chromosome VI

51. Elliott, K., Bostrom, M., Filges, S., Lindberg, M., Van den Eynden, J., Stahlberg, A., Clausen,

52. Pich, O., Muinos, F., Sabarinathan, R., Reyes-Salazar, I., Gonzalez-Perez, A. and Lopez-

53. Brown, A.J., Mao, P., Smerdon, M.J., Wyrick, J.J. and Roberts, S.A. (2018) Nucleosome

54. Jiang, Y., Zhang, N.R. and Li, M. (2017) SCALE: modeling allele-specific gene expression by single-cell RNA sequencing. Genome Biol, 18, 74.

55. Heffernan, T.P., Simpson, D.A., Frank, A.R., Heinloth, A.N., Paules, R.S., Cordeiro-Stone, M.

56. Li, W., Hu, J., Adebali, O., Adar, S., Yang, Y., Chiou, Y.Y. and Sancar, A. (2017) Human

57. Hu, J., Li, W., Adebali, O., Yang, Y., Oztas, O., Selby, C.P. and Sancar, A. (2019) Genome-

58. Martin, M. (2011) Cutadapt removes adapter sequences from high-throughput

59. Li, H. and Durbin, R. (2009) Fast and accurate short read alignment with Burrows-Wheeler

60. Li, H., Handsaker, B., Wysoker, A., Fennell, T., Ruan, J., Homer, N., Marth, G., Abecasis, G.,

61. Giusti-Rodríguez, P., Lu, L., Yang, Y., Crowley, C.A., Liu, X., Juric, I., Martin, J.S., Abnousi, A., Allred, S.C. and Ancalade, N. (2019) Using three-dimensional regulatory chromatin interactions from adult and fetal cortex to interpret genetic results for psychiatric disorders and cognitive traits. bioRxiv, 406330. 


\section{FIGURE \& TABLE LEGENDS}

668 Figure 1. Excision repair kinetics and early-repair hotspots in normal human

669 fibroblasts. (A) Experimental design to measure DNA damage and excision repair of the

670 UV-induced (6-4)PP and CPD across different timepoints. (B) Detection of excision 671 products at 0 min and 2 min timepoints in vivo. Following UV irradiation, the excised

672 oligonucleotides were purified by TFIIH immunoprecipitation, radiolabeled, and resolved

673 in a 10\% sequencing gel. DNA excision products of (6-4)PP can be detected as early as

6742 min upon damage induction. (C) Principal component analysis of genome-wide excision

675 repair as measured by XR-seq shows repair kinetics across different timepoints. Between

$6761 \mathrm{~min}$ and $5 \mathrm{~min}$, excised oligonucleotides were not degraded, and thus XR-seq measured

677 cumulative repair. (D) Each row is a sample, and each column is a specific total number

678 of reads per genomic bin. The color in the heatmap corresponds to the log counts of the

679 number of bins with specific read depths. Early-repair hotspots exist in samples collected 680 at early timepoints.

682 Figure 2. Distribution of DNA damage, repair, and epigenomic markers at identified 683 repair hotspots and coldspots. XR-seq and Damage-seq data are shown for both 684 strands, marked with + and -. Epigenetic data from ChIP-seq of histone modifications and 685 DNase-seq are plotted on the same scale for cross comparison. Read count data 686 normalized by sequencing depth are visualized in the Integrative Genomics Viewer. (A) 687 A (6-4)PP repair hotspot from chr2. (B) A (6-4)PP repair coldspot from chr1. (C) A CPD 688 repair hotspot from chr1. All examples are from intronic gene regions overlapping 689 annotated enhancers. Zoomed-in view of canonical sequences is overlaid in the bottom, 690 with the damage/repair sites shown in dashed boxes.

692 Figure 3. Repair hotspots are not hypersensitive to ultraviolet radiation. There is no 693 enriched DNA damage, as measured by Damage-seq, adductSeq, and CPD-seq, at the 694 identified repair hotspots for (6-4)PP and CPD. (A) Read counts for DNA damage are 695 computed in repair hotspots, coldspots, and random spots. The regions corresponding to 696 the different repair categories are extended at both ends for $20 \mathrm{bp}$ and $500 \mathrm{bp}$, respectively, 697 to account for the shallow sequencing depth by quantifying DNA damage. 
699 Figure 4. Genome-wide repair hotspots are associated with epigenomic markers.

700 Chromatin accessibility (DNase I hypersensitivity) is higher for repair hotspots and lower

701 for coldspots. Repair hotspots are also characterized by higher ChIP-seq signals for

702 H3K4me1, H3K4me3, and H3K27ac, markers for gene activations.

Figure 5. Genome-wide repair hotspots are enriched for enhancers. Annotations for

(A) chromatin states, (B) CpG islands, and (C) genic/intergenic regions are shown for repair hotspots for (6-4)PP (top) and CPD (bottom), separated by strands. Repair

707 hotspots are enriched for enhancers and promoters, which are in open-chromatin regions.

708 Repair hotspots are also enriched in inter-CpG and intronic regions, both of which are

709 AT-rich.

Figure 6. Genome-wide repair hotspots overlap with FIREs and super-enhancers

712 identified by Hi-C. FIREs and super-enhancers were identified and annotated using Hi-

713 C data from human fibroblasts. Repair hotspots overlap with (A) FIREs and (B) super-

714 enhancers with significantly higher proportions compared to the genome-wide averages.

715 The solid density curves are generated from bootstrapping different regions along the

716 genome as the null case; the dashed vertical lines are the observed proportions for the

717 repair hotspots and coldspots. (C) Repair hotspots have a significantly higher number of

718 significant interactions, identified by Hi-C. (D) Two examples of (6-4)PP repair hotspots

719 (chr8:13224201-13224300 and chr14:61994601-61994700) that overlap with both FIREs

720 and super-enhancers, and loop to different regions of the genome. Hi-C data has low

721 resolution, and the significant interactions are drawn from the center of each bin, which

722 does not exactly overlap with the identified hotspot shown in red.

724 Figure 7. Genome-wide repair hotspots are enriched for early-replication domains.

725 Replication timing domains were identified using Repli-Seq data. Compared to genome-

726 wide average (random), there is a significantly higher proportion of the early-repair

727 hotspots located in the early-replication domains. 
bioRxiv preprint doi: https://doi.org/10.1101/2020.04.16.045369; this version posted September 11, 2020. The copyright holder for this preprint (which was not certified by peer review) is the author/funder, who has granted bioRxiv a license to display the preprint in perpetuity. It is made available under aCC-BY-NC 4.0 International license.

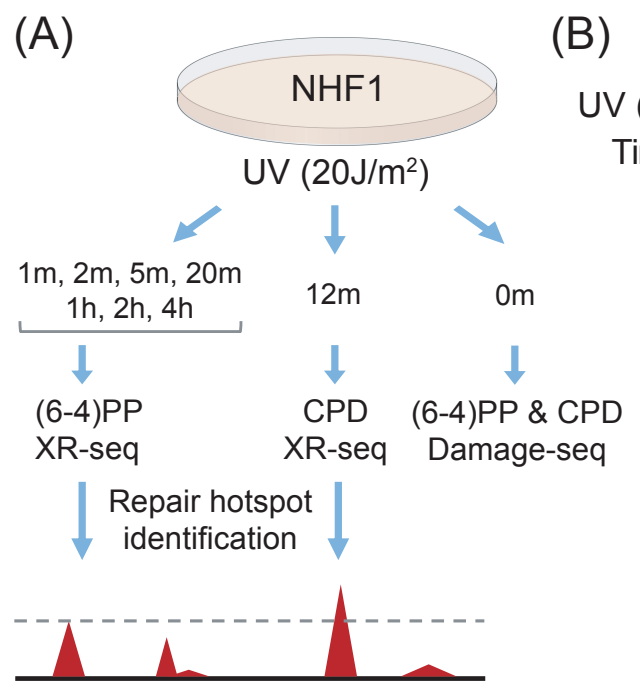

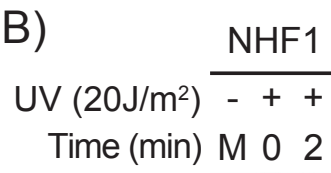

50 nt -

17nt -
(C)

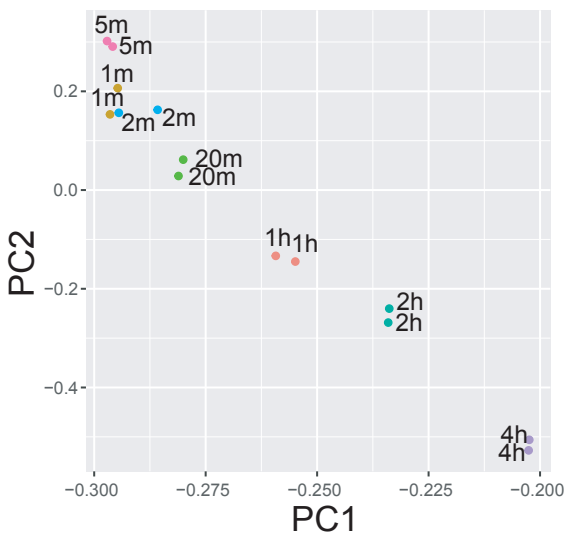

(D)
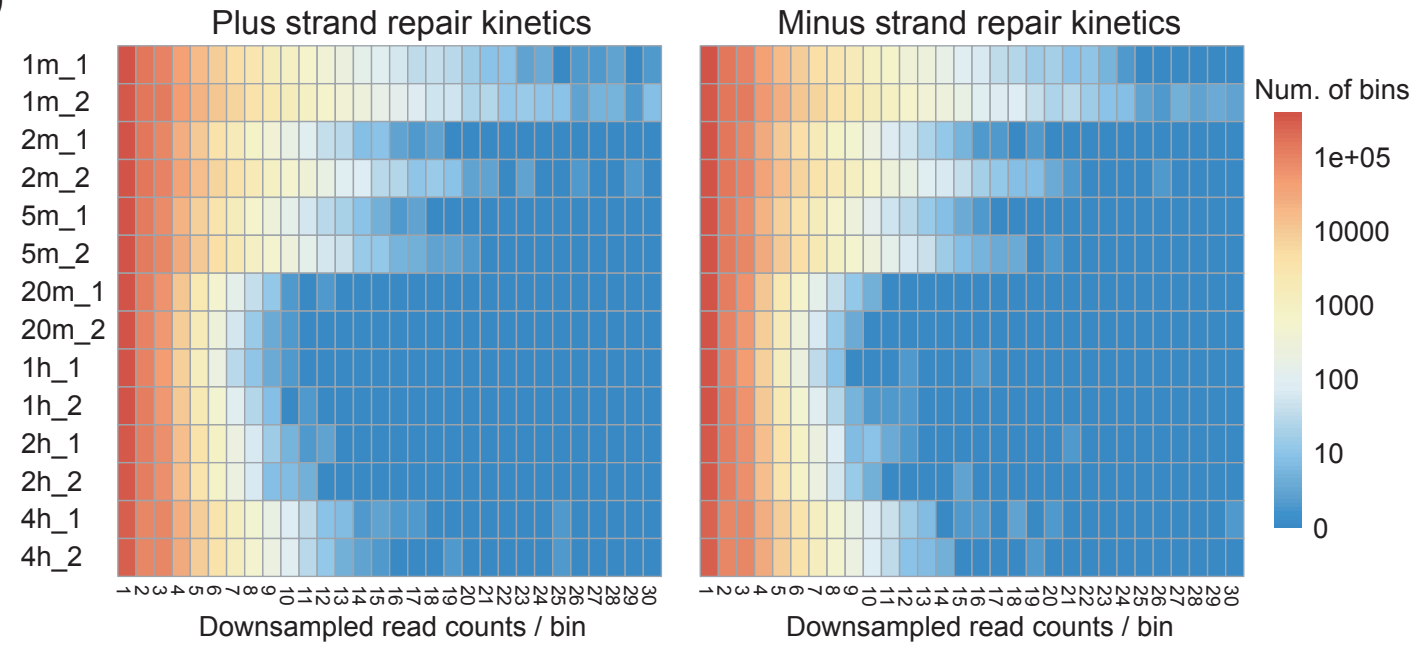
bioRxiv preprint doi: https://doi.org/10.1101/2020.04.16.045369; this version posted September 11, 2020. The copyright holder for this preprint (which was not certified by peer review) is the author/funder, who has granted bioRxiv a license to display the preprint in perpetuity. It is made available under aCC-BY-NC 4.0 International license.

(A)

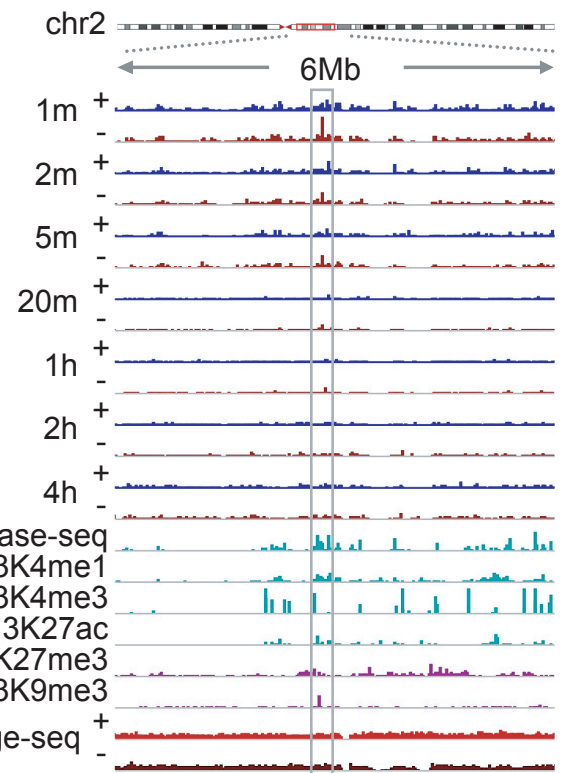

Damage-seq

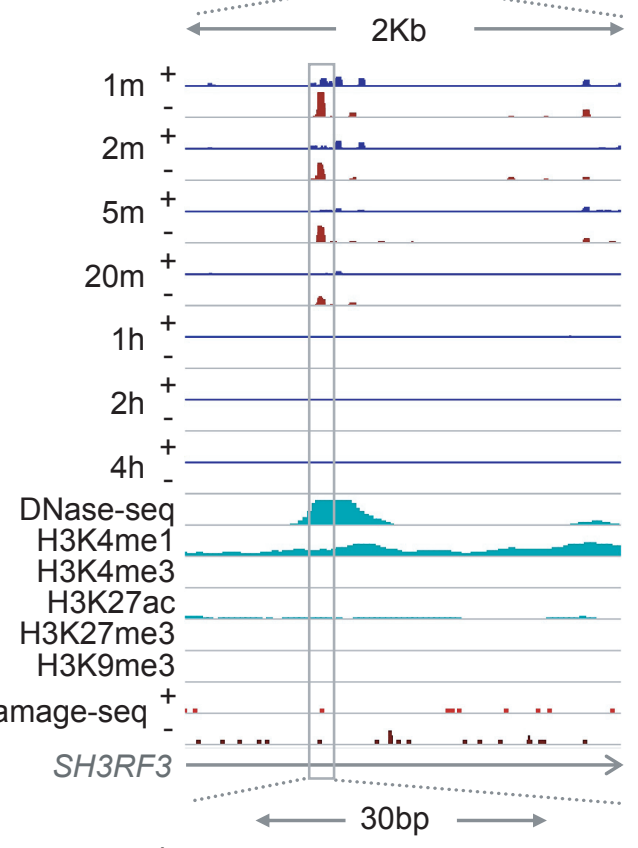

Sequence + - СACTACTTACCTCG̈T̈̈̈CTCTCACGAGTGTCCTACCGGAGTGAGGTG
(B)

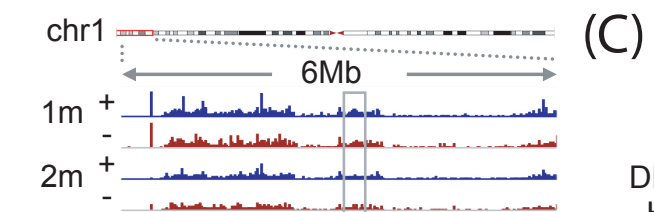

(C)
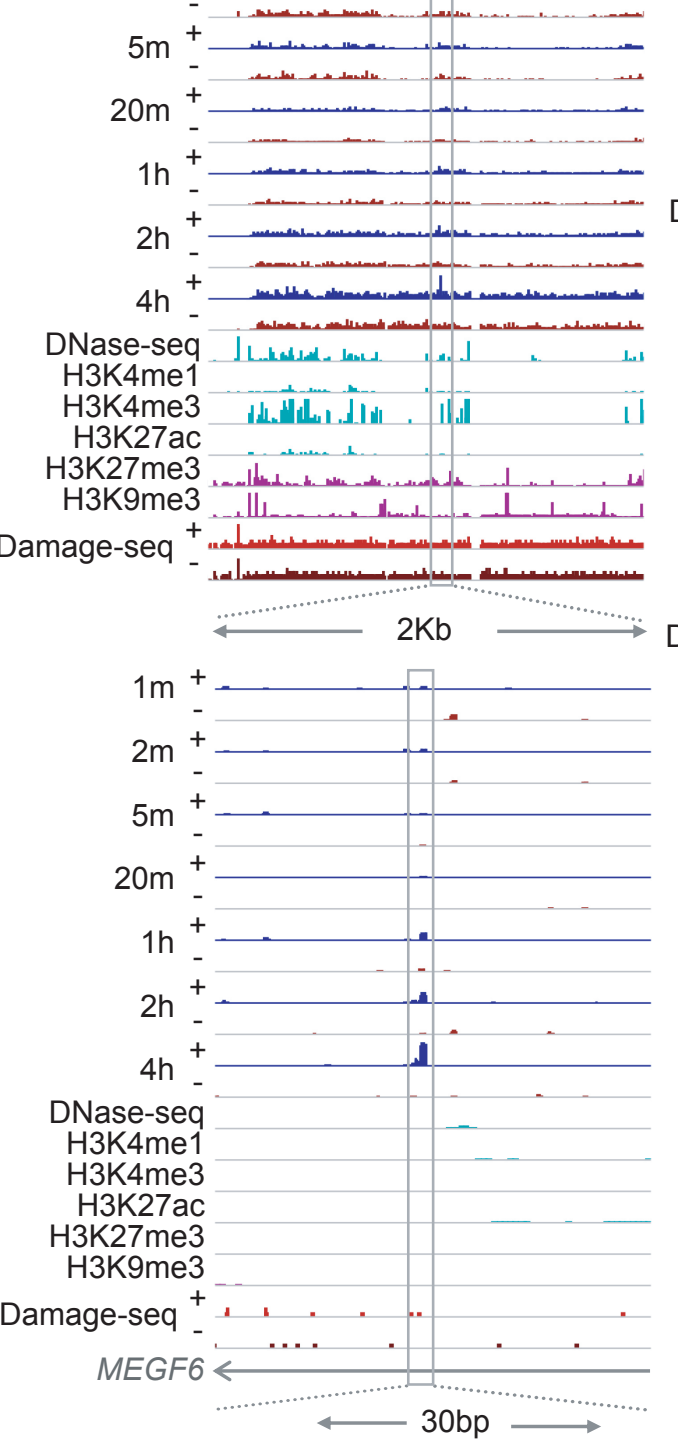

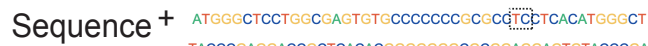

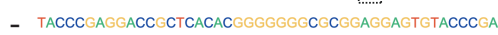

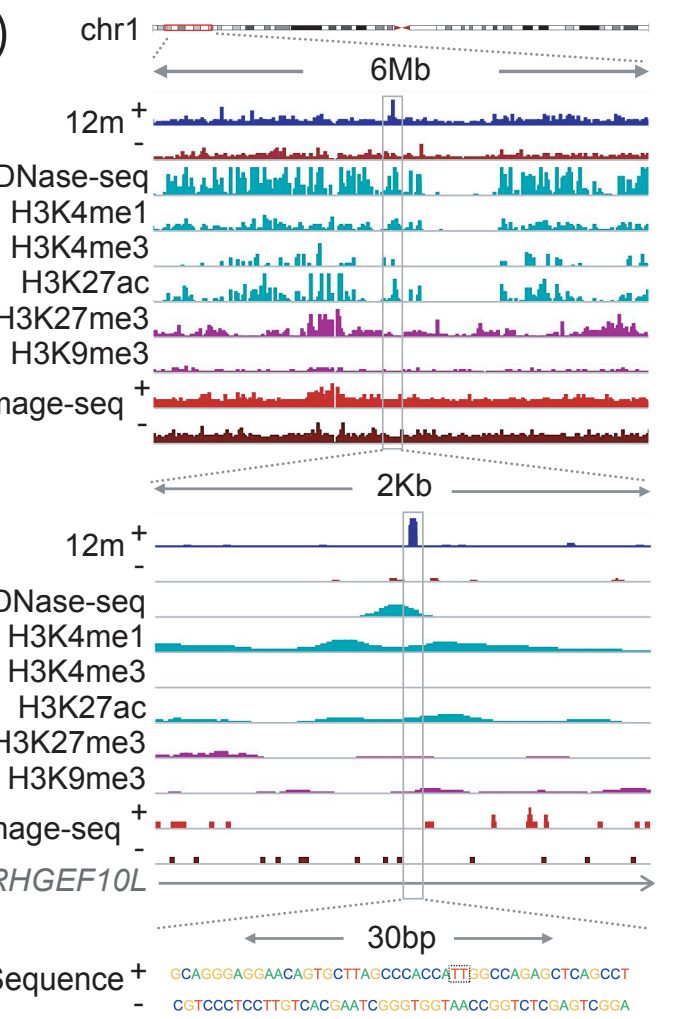

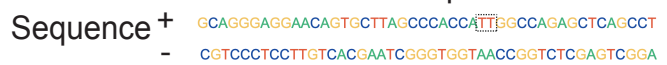


bioRxiv preprint doi: https://doi.org/10.1101/2020.04.16.045369; this version posted September 11,2020 . The copyright holder for this preprint (which was not certified by peer review) is the author/funder, who has granted bioRxiv a license to display the preprint in perpetuity. It is made available under aCC-BY-NC 4.0 International license.

(A) (6-4)PP Damage-seq

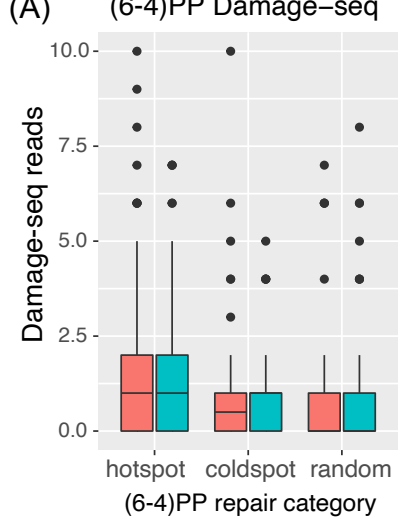

(B)

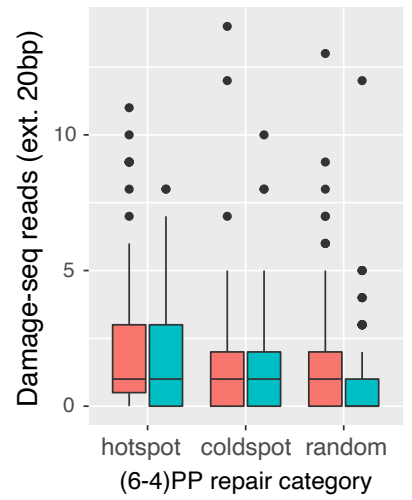

(C) (6-4)PP Damage-seq

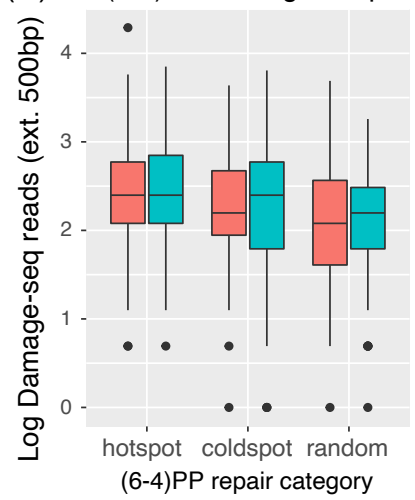

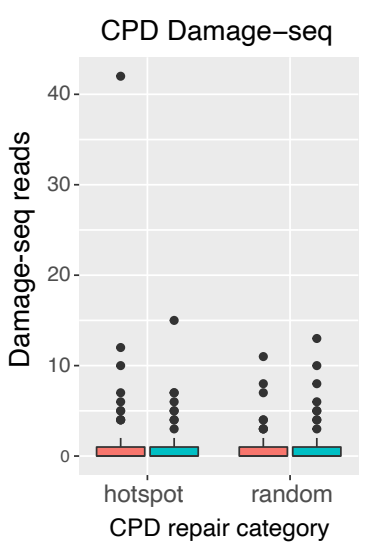
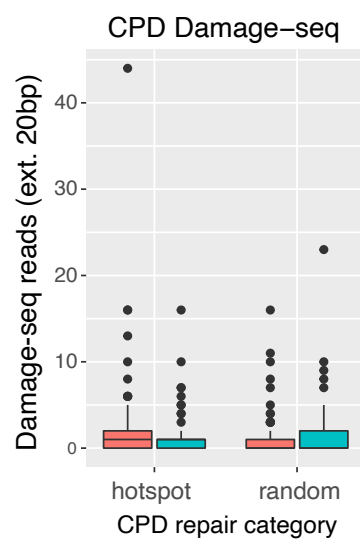

CPD Damage-seq

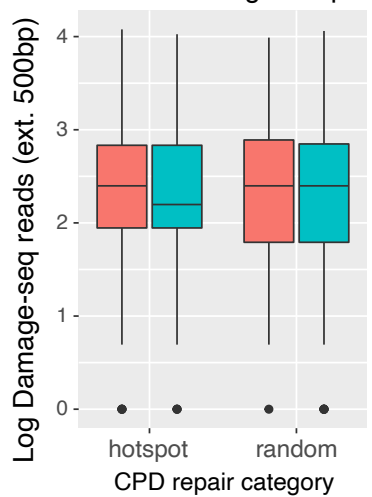

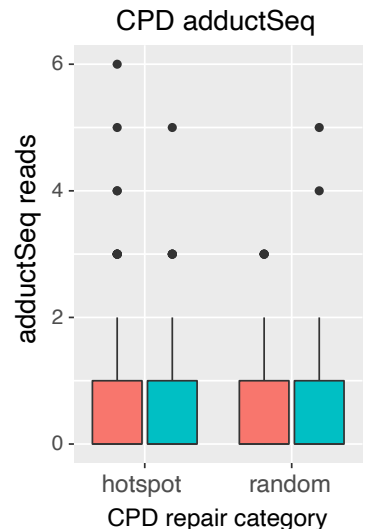
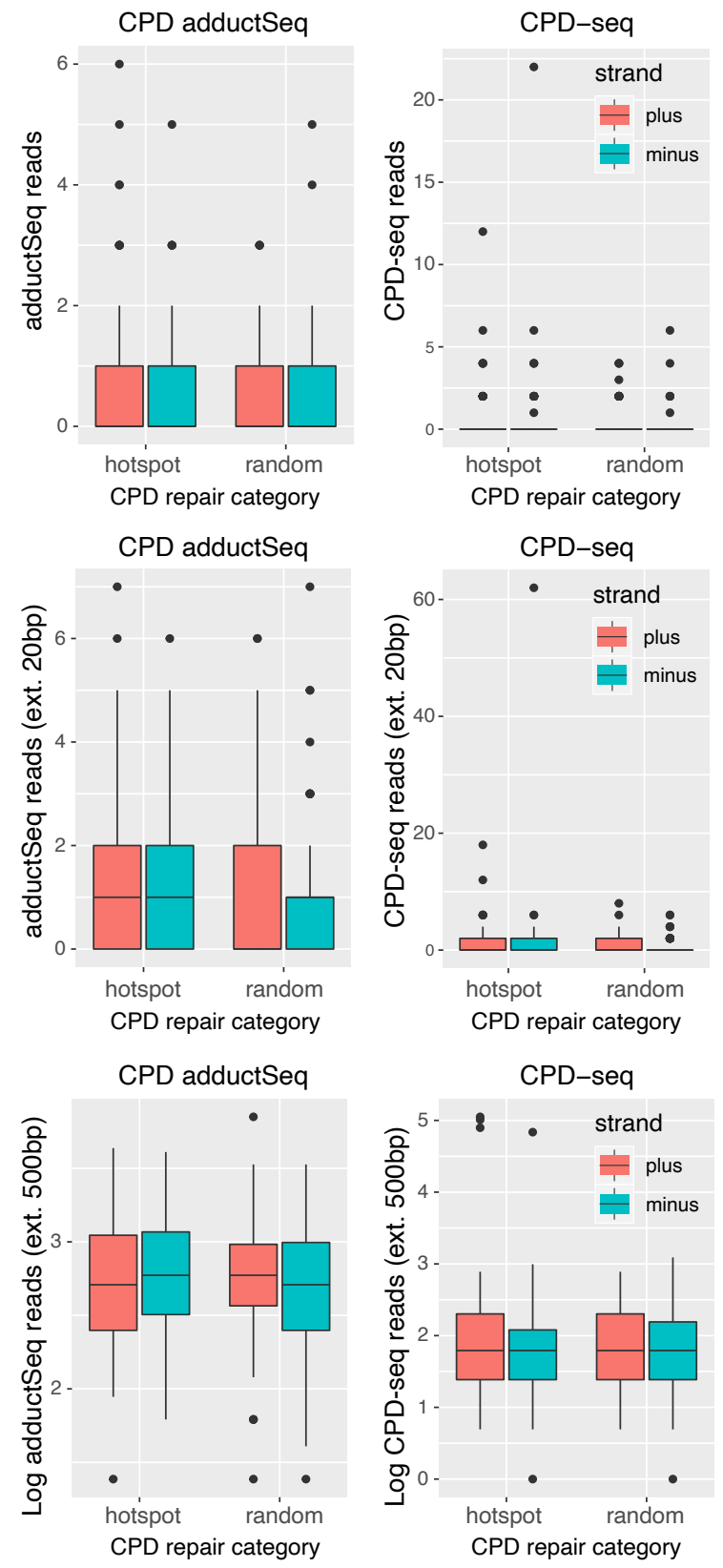
bioRxiv preprint doi: https://doi.org/10.1101/2020.04.16.045369; this version posted September 11, 2020. The copyright holder for this preprint (which was not certified by peer review) is the author/funder, who has granted bioRxiv a license to display the preprint in perpetuity. It is made available under aCC-BY-NC 4.0 International license.

(A) DNase I hypersensitivity

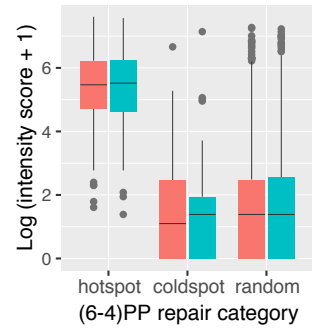

(B) DNase I hypersensitivity

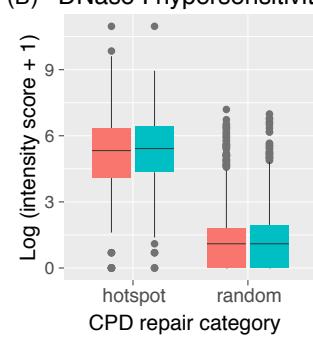

H3K4me1

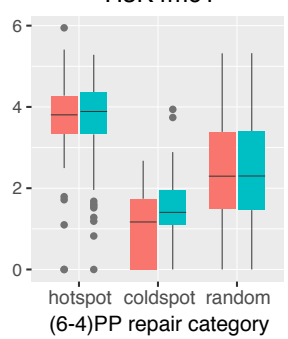

H3K4me1

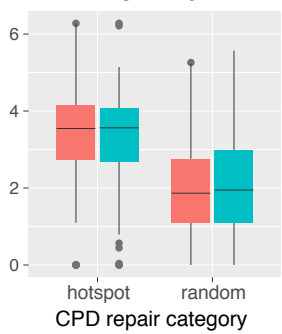

H3K4me3

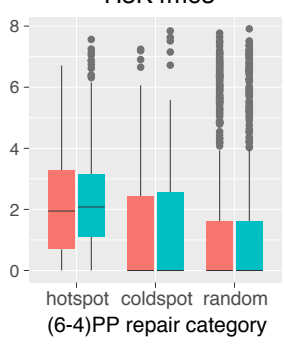

H3K27ac

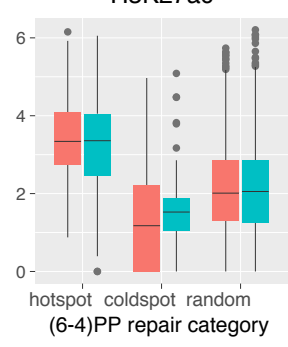

H3K27me3

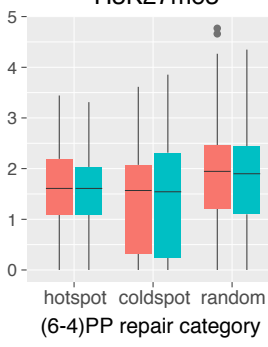

H3K27me3

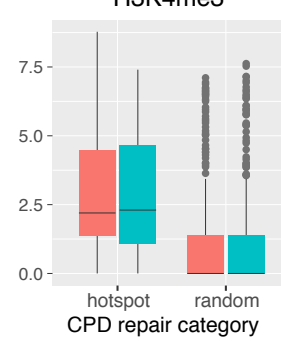

H3K27ac

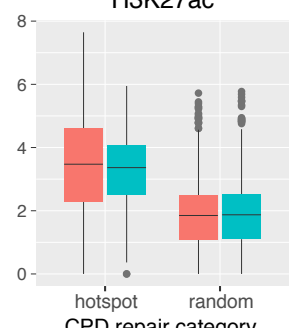

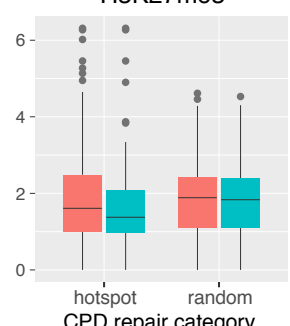

H3K9me3

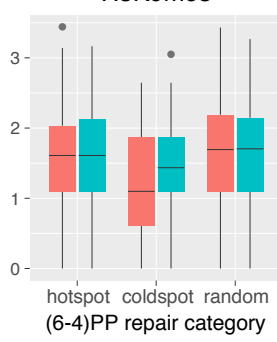

H3K9me3

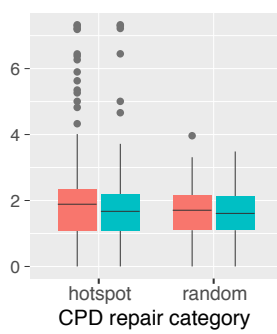

strand

$\frac{1}{4}$ plus

minus

strand

$\frac{1}{1}$ plus

minus 
(A)
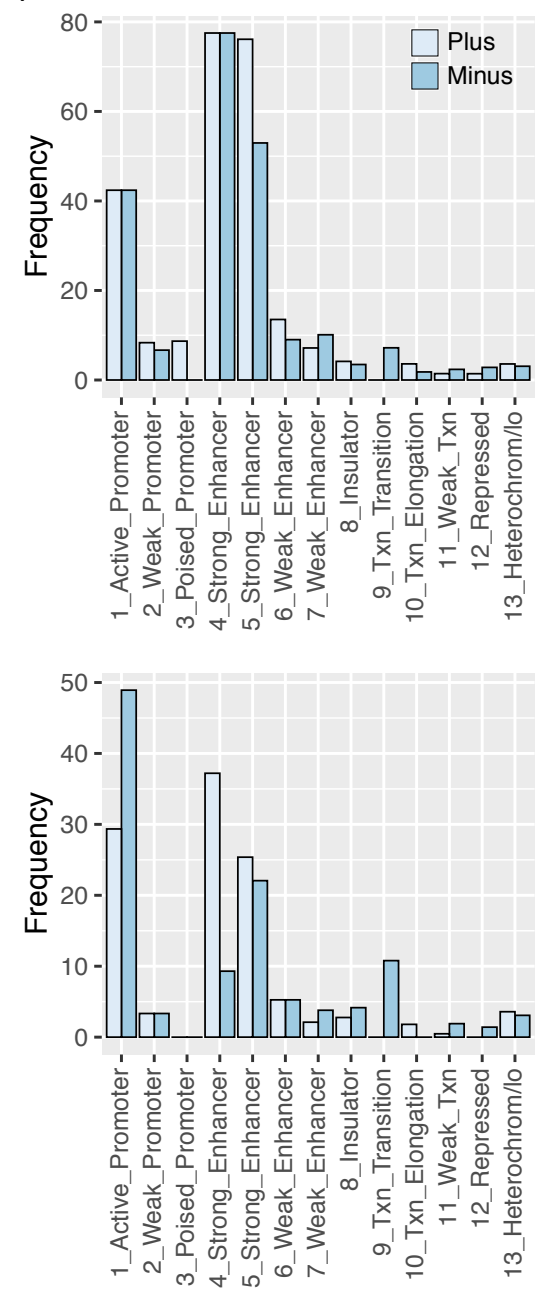

(B)
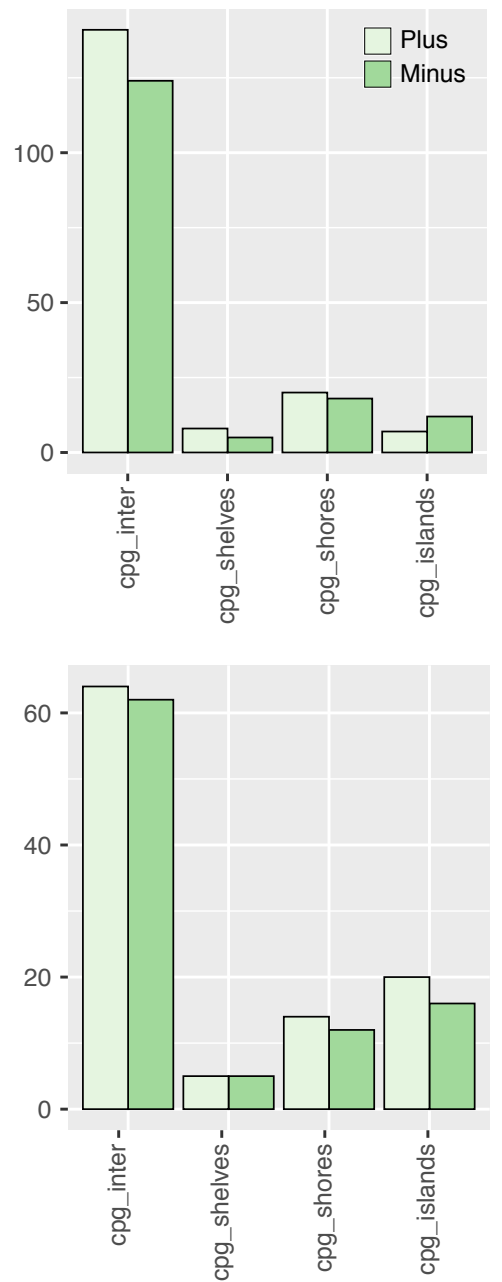

(C)
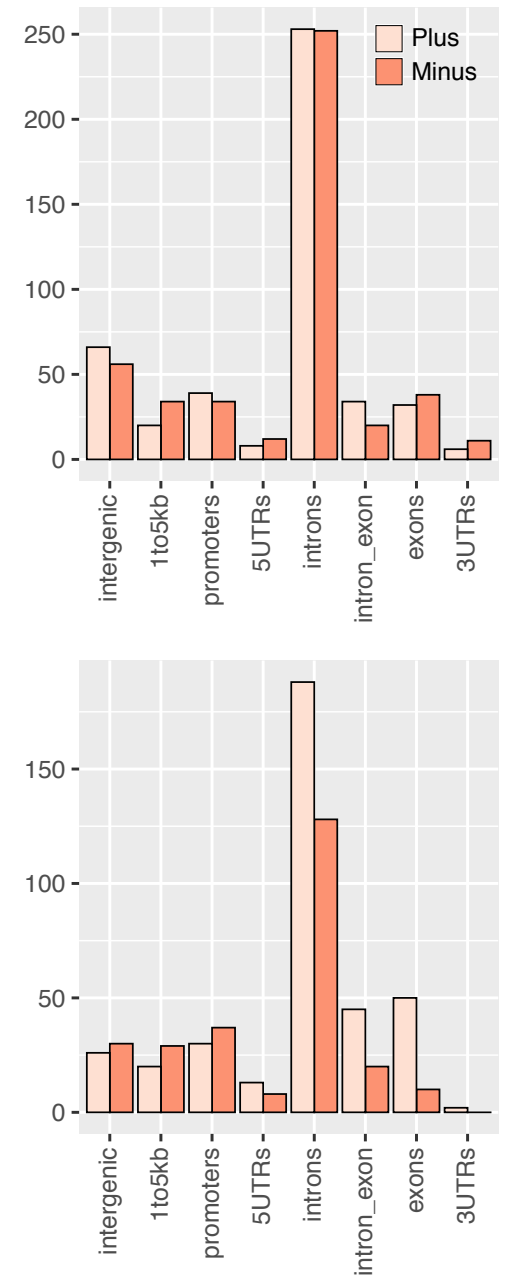
(A)

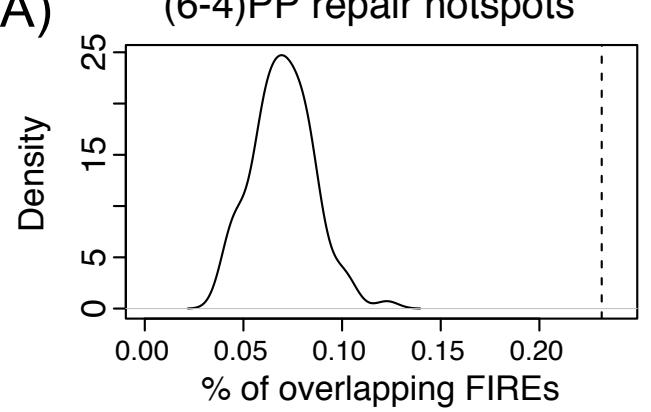

(B)

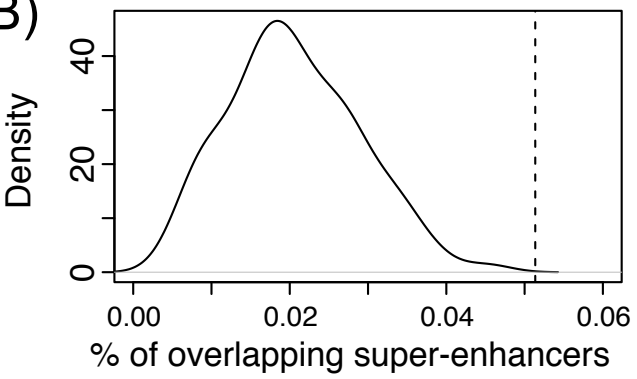

(6-4)PP repair coldspots
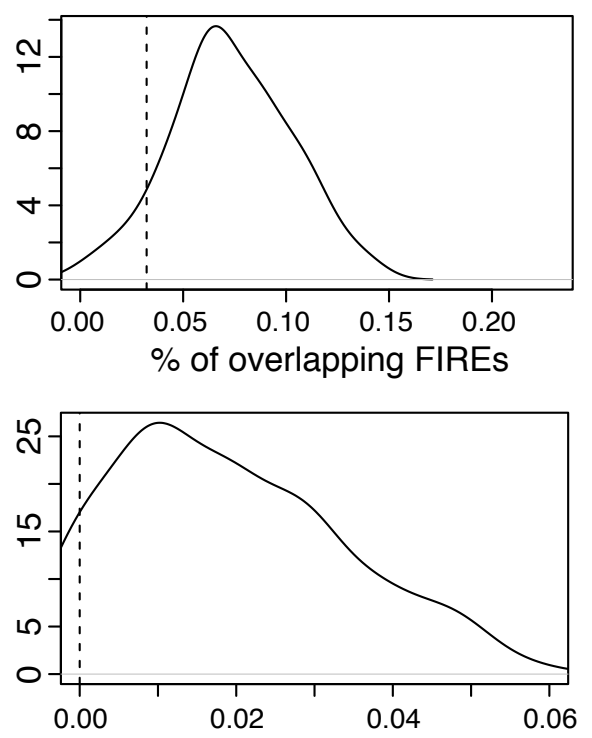

$\%$ of overlapping super-enhancers
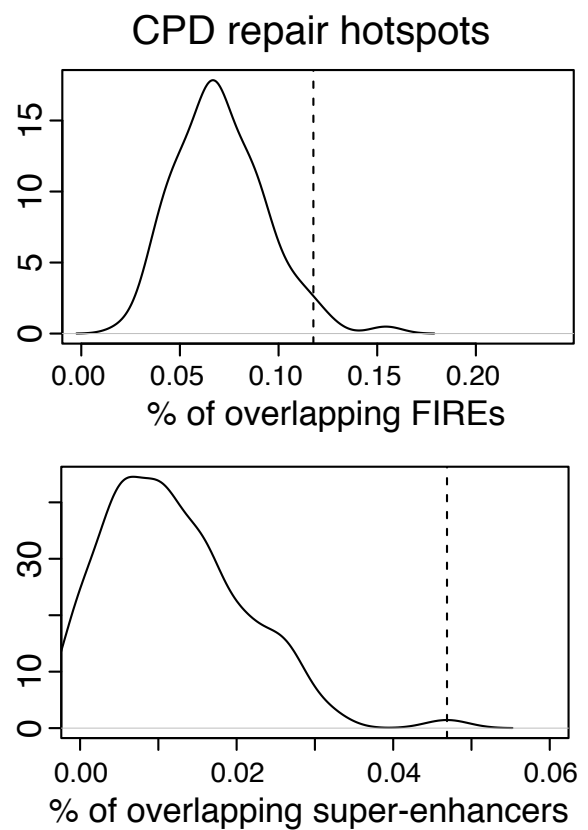

(C)

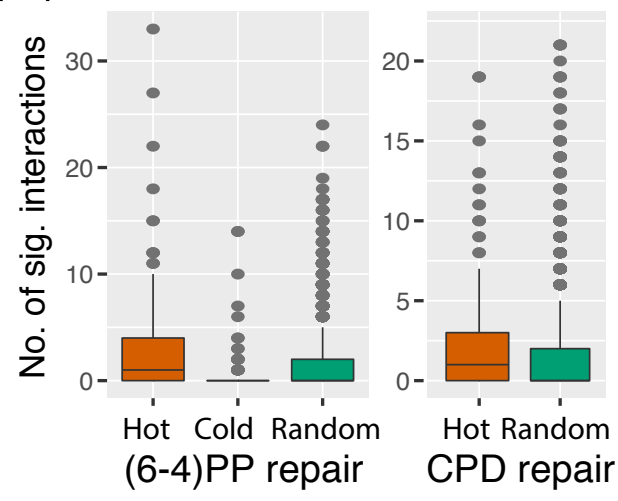

(D)

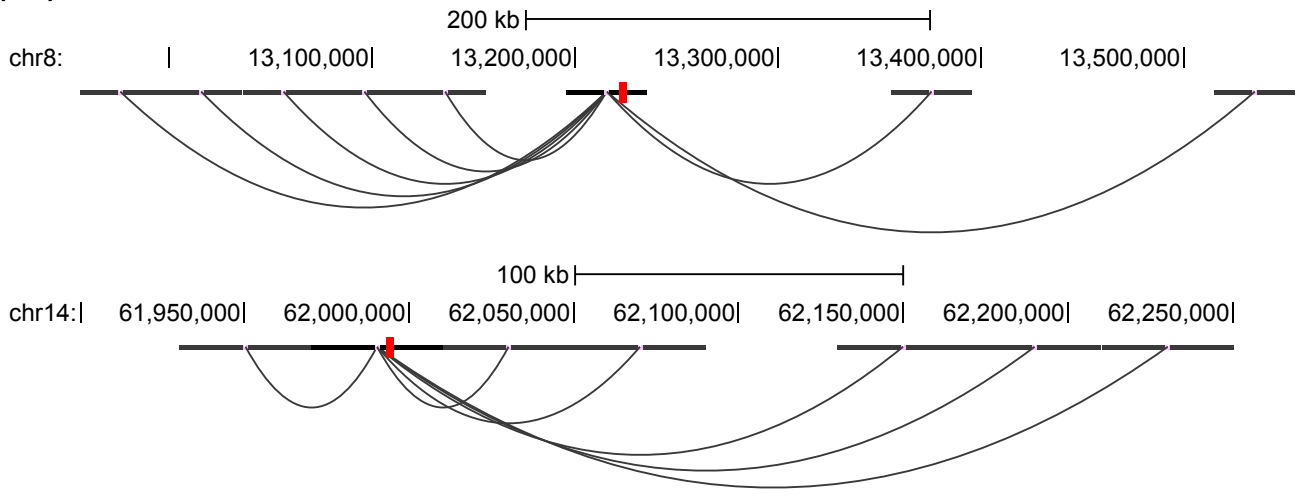


bioRxiv preprint doi: https://doi.org/10.1101/2020.04.16.045369; this version posted September 11, 2020. The copyright holder for this preprint (which was not certified by peer review) is the author/funder, who has granted bioRxiv a license to display the preprint in perpetuity. It is made available under aCC-BY-NC 4.0 International license.

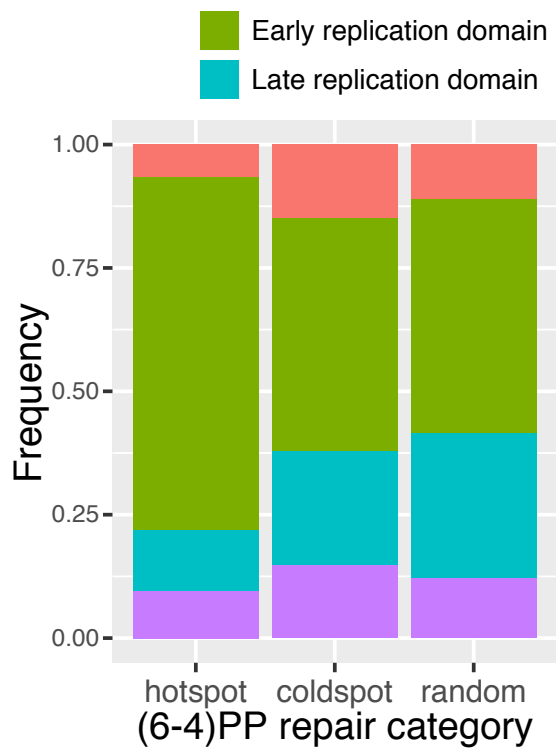

Down transition zone

Up transition zone

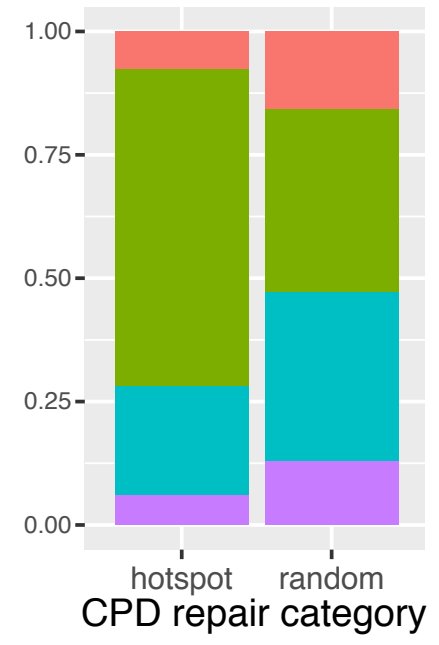

Figure 7 